\title{
Radiation Source Localization based Genetic and Fuzzy Agents using Robotics Wireless Sensor Networks
}

\author{
Amira A. A.Elmagid \\ Physics Department, \\ Faculty of Girls Collage for \\ Science, Art and Education \\ Ain Shams University \\ Cairo- Egypt
}

\author{
S. M. El-Ghanam \\ Electronics lab \\ Physics Department \\ Faculty of Girls for Science, Art \\ and Education, \\ Ain Shams University \\ Cairo-Egypt
}

\author{
Rabie A. Ramdan \\ Computer Engineering \\ Department \\ Faculty of Engineering \\ Cairo University \\ Giza-Egypt
}

\author{
Mahmoud E.Marie \\ Systems and Computer \\ Engineering Department \\ Faculty of Engineering \\ Alazhar University, \\ Cairo-Egypt
}

\author{
Shahinaz M. Al-Tabbak \\ Computer Applications Group, \\ Physics Department \\ Faculty of Girls for Science, Art \\ and Education \\ Ain Shams University \\ Cairo- Egypt \\ S.A. Kamh \\ Electronics lab \\ Physics Department \\ Faculty of Girls for Science, Art \\ and Education \\ Ain Shams University \\ Cairo-Egypt
}

\begin{abstract}
The recent increasing threat of radiological weapons technologies has highlighted the need for superior detection of hazardous emission sources. One promising area of technological development is radiation source detection using tracing mobile robot. In this paper, a novel algorithm based on GA is proposed for localization problem of such dangerous source using single robot. In which, if the estimated source location is gathered, the algorithm process is being terminated. The adaptive GA based on fuzzy logic is also introduced for comparison. Simulation results indicate that the proposed adaptive genetic algorithm have a better performance and faster than GA. In addition, the paper presents an investigation of radiation source localization by addressing the explanation of two novel algorithms that are assumed when considering group of autonomous mobile robots system.
\end{abstract}

\section{General Terms}

GA, Fuzzy Logic,WSN

\section{Keywords}

Keywords are your own designated keywords which can be used for easy location of the manuscript using any search engines.

\section{INTRODUCTION}

The interest of identification radiation sources has been increased dramatically as a part of the defense strategy against radiological terrorism scenarios [1]. The focus of the challenge is on confronting the radiological aggressions, which may be performed using radiological dispersion devices or improvised nuclear devices such as "dirty bombs" that pose a serious bioterrorism threat. The possibility of identifying the traces of such sources enables the user to detect them before they are sitting to operate, while they are being transformed or stocked.

Absolutely, when the radiation accident happens, there is a massive harmful radiation region. The major hazards to people in the vicinity of the plume are radiation exposure to the body from the cloud and particles deposited on the ground, inhalation of radioactive materials, and ingestion of radioactive materials which usually characterized by a plume region. This could be taken action by inhalation of radioactive gases, ingestion of radioactive materials and particles deposited on the ground [2], [3].

Increasing threat of radiological aggressions has highlighted a considerable concern in research on confrontation such attacks. Based on such a technological vision, it must be able to encountering the widespread threats that target the civilian in both private and public places. There are different detection and localization methods including the one that uses wireless sensor network (WSN).

Recently, with the remarkable advances of microelectronics fabrication technology, there has been increased interest in WSNs due to the promised glaring future and Advancements to the world of information technology [4] , [5]. WSN mainly consists of distributed number of small, low priced, and limited energy sensor nodes with restricted capabilities of computing and communicating ranges [6]. Nodes in WSNs cooperatively form an ad hoc wireless network to monitor physical phenomena of the monitored field [7]. Accordingly, the radiation detection domain has been largely evolved by dint of recent advent of sensor network technologies, and also for deducing the source traces by utilizing the measured data gathered from the multi sparse sensors.

Consequently, WSN radiation detection system can read the monitoring data, and analyze the radiation information transmitted by each node. If the radiation on certain position surpasses the normal value, the system sends out the corresponding alarm message according to the source address and realizes the real time warning. The ability to rapidly localize a radiological source can assist emergency responders to disable, isolate and safely remove such source [8][8]. One of the most considerable safest ways to accomplish this task is using robots.

Today, robot system is often the safest way to enter a very high radiation area. Their main task is to perform a repetitive mission or to execute dangerous tasks that are risky to human. Furthermore, robots could be designed for exceptional 
utilization which means that they are not work consistently but rather only when they are being in demand. Noting that, these robots are required for being equipped with some of the sensors that can be used for such applications. One of the new smart devices that can be used as sensors is the Intelligent Personal Radiation Locator (IPRL) [9]. Such device is small in size that can be carried on a robot. It also has the capability of wireless communication with similar devices as well as with others that might be used as data loggers or aggregators.

The contributions in this paper are two folds; first, the problem of radiation source localization is investigated. A radiation source such as Cesium-137 or Cobalt-60 is placed into 2-D area. Since human safety is one of the proposed work major concerns, a single robot equipped with IPRL sensors tries to reach the radiation source location by moving towards it. Two of the efficient techniques were utilized which are Genetic Algorithms $(G A)$ and Fuzzy Logic $(F L)$. $F L$ is used along with $G A$ to enhance its performance. The $G A$ and $F L$ are used to guide the robot to the radiation source location. The second contribution is the study of radiation source localization problem using multiple robots. For efficient solution to this problem, two new algorithms - based on adaptive $G A$ and $F L$ were proposed.

\section{OVERVIEW}

In most cases, Photons that are generated by a radiation source follow Poisson distribution. Consider a fixed radiation source and a fixed detector where the rate at which the detector records photons from the source is $\lambda$. In such case, the probability of detecting the radiation source is [10]:

$$
P(n \mid \lambda, \Delta t)=\left(\frac{(\lambda \cdot \Delta t)^{n}}{n !} \cdot e^{-\lambda \Delta t}\right.
$$

where $\mathrm{n}$ is the number of hits recorded by the detector in time interval $\Delta t$.

Radiation detection using wireless sensor networks has been also a topic of interest to many researchers including [11] and [12] . For instance in [11], sensors are used to detect radiation in high energy physics labs. Sensor grid architecture is suggested according to the feature of high energy physics environment and sensors are manually placed in their appropriate places. In[12], the authors designed a WSN to address the problem of low anti-interference ability, timeconsuming network construction and high energy consumption occurring in existing system.

Qing-Hao Meng et. al. [13] solved the mobile robot odor localization (MROL) problem using an improved Ant Colony Algorithm (ACA). The so-called MROL means localizing an odor source with mobile robots. The algorithm was realized through three phases, which are genetic algorithms (GAs) based local search, global search, and pheromone update. The GA ensured that the optimal or sub-optimal points could be found within local areas. The global search phase consisted of random and probability based searches. The random search could prevent the ACA from getting into local optimum. Two Gaussian concentration models were used to describe the odor distribution [13].

Adam T. Hayes et. al. performed number of investigations in [14], [15], [16] that solve an odor source localization by using multi robots by implementing distributed algorithm where multi robots performance could surpass single robot efficiency. They have proved that the real robot could properly localize an odor source based on subtask and plume traversal concept. Although the multi robots concept was applied, the collaboration technique between robots is too poor where the full use of robots information about odor intensity is retained for itself, instead of being participated to the others. Applying the proposed algorithm raises the possibility that more than one robot could search in the same area repeatedly.

\section{PROBLEM FORMULATION}

In this section, the radiation source detection problem is explained. In this problem, a monitored space $A$ is described as a 2-D environment. $A$ is assumed to be modeled as oblong bounded with $L$ length and $W$ width measured in arbitrary units. $A$ is virtually divided into grid of cells indexed with a where $a=1, \ldots A$. In addition, it is assumed that the static point radioactive source of unknown strength $A_{0}$ is located in the predefined surveillance area $A$. Such a source might be corresponding to a dirty bomb left at a concealed location in airport, train station or other public venues. The unknown location of the source is given by $\left(x_{s}, y_{s}\right)$ within the boundaries of $A$. The source induces a radioactivity of intensity $\mathrm{I}(\mathrm{x}, \mathrm{y})$ at any location $(\mathrm{x}, \mathrm{y}) \in A$. Also, a single or multiple robots $S_{i}$ with IPRL radiation detectors which can be located anywhere in the monitored field denoted by $\left(\mathrm{x}_{\mathrm{i}}, \mathrm{y}_{\mathrm{i}}\right) \in$ $A$. Background radiation is universally present, and its measurement by $S_{i}$ is denoted by $B_{i}$. Actual radiation sensors, however, may have different sensitivities, and their readings may differ significantly even when they are subjected to the same level of radioactivity. virtually, this is strongly depending on the sensor efficiency $E_{i}$ where it is assumed to be constant where $t$ is empirically determined with a calibration experiments.

Consequently, the reading measured by sensor $S_{i}$ could be computed by equation (2) [17]:

$$
I_{i}=\frac{2.22 \times 10^{6} \times A=E_{i}}{\left(x_{s}-x_{i}\right)^{2}+\left(y_{s}-y_{i}\right)^{2}}+B_{i}
$$

Based on this scenario, the probability of locating the sensor in cell $k$ at time $t$ is assumed $p_{k}^{t}$. Therefore, the overall probability of finding the radiation source in the monitored field could also be determined by equation (3):

$$
\forall t \sum_{k=1}^{K} p_{k}^{t}=1
$$

Based on the previous information about the radiation source and the radiation field as well as the robot(s) position(s), the problem is how efficiently the robot can find the radiation source.

\section{PROPOSED SOLUTIONS}

This section presents our proposed solution for the radiation source localization. Our proposed methods focus on directing the single and/or multi-robots to the radiation source with minimum moving distance. It is assumed that the monitored area $A$ is divided into grid of cells (zones) with length $L_{i}$ and width $W_{i}$. The first subsection explains the GA while the following subsection includes the fuzzy-rule-based selfadaptive genetic algorithm solution. In addition, two variations of the multi-robots solution are presented.

\subsection{Genetic Based Solution}

Genetic Algorithms (GA) is one of the most powerful heuristics for solving optimization problems where the theoretical foundations of GAs were originally developed by Holland [18][18], [19]. The idea is based on the mechanics of 
natural selection and inspired by the biological mechanisms of evolution and heredity. In GA, chromosomes are designed to describe a feasible location scheme for the available robot(s). The length of each chromosome (number of genes) is considered to be equal to the coordinators of robot including its location $(\mathrm{x}, \mathrm{y})$ corresponding to the given monitored field. It is also assumed that, if a robot is deployed in zone $a \in A$, the gene represents the location of the robot is restricted by its zone borders. Fig. 1 illustrates the structure of a chromosome used to represent simple example for the robot located at point $(3,7)$ in zone dimension of $x=0$ to 10 and $y=0$ to 10 .

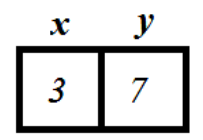

Fig. (1): Example of a chromosome for the robot position.

The initial population is generated randomly and crossover and mutation operators are applied on the generated $n$ chromosomes. The fitness of each generated chromosomes is measured using the function given in equation (1).

Since the GA optimization procedure highly depends on the crossover and mutation methodologies, the crossover methodologies used in this paper are based on the heuristic functionality. The heuristic crossover operator uses fitness of the parents for leading the search process towards the most promising zones. It returns a child that lies on the line containing the two parents, a small distance away from the parent with the better fitness value (BestParent) and in the direction away from the parent with the worst fitness value (Wors Parent). The distance between the child from a better parent could be specified by the parameter Ratio. The default value of Ratio ( $\mathrm{r}$ ) is assumed 1.2. When considering two chromosomes to be crossed, then the offspring are created according to the following equations:

Offspring $=$ BestParent $+\mathrm{r} *($ BestParent - WorstParent $)(4)$

At the same time, the mutation methodology used in this paper is based on a uniform distribution method. The algorithm selects a fraction of the vector entries of an individual for mutation, where each entry has a probability rate of being mutated. The algorithm replaces each selected entry by a random number selected uniformly from the range of that entry. The new gene value is skipped if it falls outside of the user-specified lower or upper bounds for that gene. The new generated position is considered as the new position of the robot that moves to and starts a new iteration. As can been recognized, the small the cell size, the fast the GA reaches the border of the cell. Certainly, the radiation source and its strength based on equation (2) play the main role in directing the robot and leading the GA in the right direction.

\subsection{Self-Adaptive Genetic Based Fuzzy Logic Solution}

Fuzzy logic is almost synonymous with the theory of fuzzy sets, a theory which relates to classes of objects with unsharp boundaries in which membership is a matter of degree. Fuzzy logic was first developed by Lotfi A. Zadeh in the mid-1960s for representing uncertain and imprecise knowledge [20]. It provides an approximate but effective means of describing the behaviour of systems that are too complex, ill-defined, or not easily analyzed mathematically. Fuzzy variables are processed using a system called a fuzzy logic controller. It involves fuzzifier, inference engine, and defuzzifier. In this section, we introduce another version of GA with the adaptation of fuzzy logic method for the radiation detection problem.

As noticed, crossover and mutation have crucial effects in chromosomes diversiform. Actually, the crossover $P_{C}$ and mutation $P_{M}$ percentages draw the line of how often crossover and mutation operators are applied to chromosomes through the evolutionary process. It is noted that, large $P C$ and $P M$ leads to more GA search effectively of the solution space, therefore the finding better solutions prospective is increased and even shooting the inclusive optimums. Despite this, for large $P_{C}$ and $P_{M}$. The risky of good chromosomes destruction and damage will increase. Consequently, the GAs solutions could be slowed down or even though preventing from convergence. During GAs optimization approach, online adaptive of $P C$ and $P M$ according to the current state of optimization can considerably enhance the solution quality and leads to rapid convergence. The chromosomes current fitness level $\left(F C_{r a t}\right)$ and the GA optimizing stage $\left(G_{r a t}\right)$ were selected to guide the adaptation of $P C$ and $P_{M}$ [21].

The chromosomes current fitness level of chromosomes, $F C_{\text {rat }}$, can be represented by the average fitness of current generation, $F C_{\text {avg }}$, and the maximum and minimum fitness by current generation, $F C_{\max }$ and $F C_{\min }$, which affect $P_{C}$ and $P_{M}$. Firstly, the value of $F C_{r a t}$ could be computed using equation (5).

$$
F C_{r a t}=\frac{F C_{\text {max }}-F C_{a v g}}{F C_{\text {max }}-F C_{\text {min }}}
$$

The $F C_{\text {rat }}$ value is an indicator of the distribution quality of the current iteration chromosomes in the search space. The large value is pointing to the evenly distribution of chromosomes. Accordingly, large $P C$ could facilitate the convergence of algorithm. On the contrary, if $F C_{\text {rat }}$ has small value, the chromosomes may be fallen in some local minima, thus a larger $P M$ is required to diversify the individuals. Nevertheless, if $F C_{\text {rat }}$ has a medium value, so medium value to both $P C$ and $P M$ will be reasonable.

The value of $G_{r a t}$ is also computed using equation (6). where $G_{C n}$ is the current generation number and is $G_{T n}$ the total number of generations. The value roughly points to the phase of optimizing phase, i.e. at the beginning or ending the optimization.

$$
G_{r a t}=\left(G_{C n} / G_{T n}\right)
$$

$\mathrm{G}_{\text {rat }}$ is close to zero (small), this means the optimization of GA is in the early stage. Thus, the relatively large $P_{C}$ and $P_{M}$ should be implemented for granted more widely search in the proposed space. If $G_{r a t}$ is large, thus the GA optimizing process is tending to be finished. So, smaller $P C$ and $P M$ are more appropriate for efficiently preserving and slightly alteration of good individuals. If $G_{r a t}$ has intermediate value between 0 and 1 (medium), then the optimization of GA is in the middle of operation. Thus, medium $P C$ and $P M$ values will be appropriate. Table 1 summarizes above analyses. 
Table (1): Fuzz y rules for the influences of key factors on PC and PM.

\begin{tabular}{c|ccc|ccc}
\hline \multirow{2}{*}{ Parameter } & \multicolumn{3}{|c|}{$\boldsymbol{G}_{\text {rat }}$} & \multicolumn{3}{|c}{$\boldsymbol{F C}_{\text {rat }}$} \\
\cline { 2 - 7 } & $\mathrm{S}$ & $\mathrm{M}$ & $\mathrm{L}$ & $\mathrm{S}$ & $\mathrm{M}$ & $\mathrm{L}$ \\
\hline $\boldsymbol{P}_{\boldsymbol{C}}$ & $\mathrm{L}$ & $\mathrm{M}$ & $\mathrm{S}$ & $\mathrm{S}$ & $\mathrm{M}$ & $\mathrm{L}$ \\
\hline $\boldsymbol{P}_{M}$ & $\mathrm{~L}$ & $\mathrm{M}$ & $\mathrm{S}$ & $\mathrm{L}$ & $\mathrm{M}$ & $\mathrm{S}$ \\
\hline
\end{tabular}

Obviously, the fuzzy logic rules are exerted from Table (1). As can be seen, the rules are very simple to be used with every generation of the GA to adjust the crossover and mutation percentages.

\subsection{Multiple Mobile Robots Behaviour for Source Localization}

A single robot system has several major benefits as low cost and easy to implement. However, its weak robustness and search capacity as well as its limited expansion seriously restrict its application in real-world problems. By contrast, a multi-robots system can overcomes the shortcomings of the single robot. In addition, the expected search time can be decreased. Certainly, the multi-robot system does not easily fall into local maxima [22].

Using multi-mobile robots indicate that the source localization process is conducted under the cooperation of a group of mobile robots. At the first time, the robots are assumed deployed randomly in the proposed area. Robots in a group should have same basic capabilities. For example, every robot in the group should have the capability to collect information. The robots should also have the capability to communicate with each other. In addition, each individual robot considers other robots in the network as sensors.

In this context, two new algorithms are being provided that integrate groups of low-cost robots, which are equipped with communication ability and relatively inexpensive sensors. Each robot acts as a node in the sensor network.

\subsubsection{The Leader Election algorithm (LEAD)}

The first algorithm (LEAD) is essentially based on the idea of leader tracking. Leader election algorithm is an approach using a leader robot to coordinate individual robots. The leader robot is elected according to the maximum recorded reading of radiation intensity. A number of tracing mobile robots collect the essential information of locations and the intensity sensor's reading values and send such information to the leader. The leader executes the adaptive $G A$ to determine the appropriate direction and accordingly moving to the selected cell. The rest of robots trace the same direction as the leader follows. This behaviour will detect the expected next cell that fits the moving guidance. Consequently, the new robot's geographical location is identified in the center of selected cell and this is one hop for each of them. This step will continue until one of the robots reaches the source location. It is worth to mention that the total number of messages that the $n$ robots send or receive in each hop equals to $2 * n-2+(n-1)$.
4.3.2. The Consultation Algorithm (CONSLUT) As for concerning the second algorithm (CONSLUT), it is mainly relies on the principle of consultation among the robots. The robots' positions and sensor's concentration values can be collected through the formed wireless network. The information will be considered as input to the FLC to generate a crisp output; the robot's optimal moving direction. In addition, in this algorithm, robots failure could be easily handled in which it is not considered in the first algorithm. As shown in Fig. (2), the FLC granted each robot the ability to create decisions of its moving direction despite of those uncertainties. In the proposed technique, the FLC is applied on each robot to generate the optimal tracking direction. Each robot will consult with other robots individually in which it can communicate by gathering the data (robot intensity reading) from and then compare it with its intensity. Depending on specific rules achieved using fuzzy logic control that are summarized in table (2), the robot decides if it will follow the consulted robot or it will decide to resolve by tracing its own action. Table 2 summarizes the rules that are subjected to the robot to make the decision. These rules are depending on the robot intensity (M. Intensity) and the consulted robot intensity (Y. Intensity). According the fuzzy controller output, the robot decides to follow its decision (Mp) or the consulted robot decision (Yp). If the robot follows the consulted robot, it will take the direction moving towards the selected robot and directly chooses its location in the center of the next provided cell. Otherwise, if the robot decision is to follow its action, it will implement adaptive $G A$ (as stated before in section 4.2.) to control moving direction to the next cell. The control algorithm is implemented on each individual robot till one of them reach near the radiation source. Based on this technique, based on this technique, it is expected that the total number of messages that the $n$ robots send or receive in each hop $=n *(n-1)$.

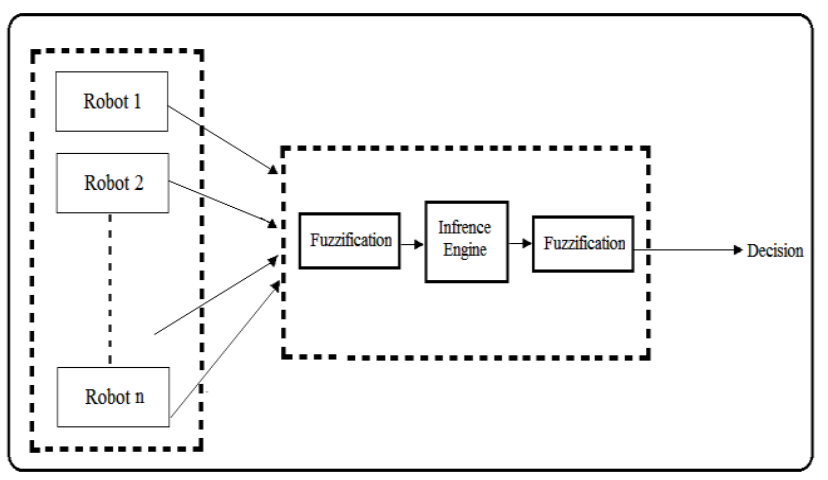

Fig. (2): Robot's Fuzzy Logic Controller.

Table (2): Fuzz y rules for the influences of key factors on Mp and Yp

\begin{tabular}{c|ccc|ccc}
\hline \multirow{2}{*}{ Parameter } & \multicolumn{3}{|c|}{ M. Intensity } & \multicolumn{3}{c}{ Y. Intensity } \\
\cline { 2 - 7 } & S & M & L & S & M & L \\
\hline Mp & S & M & L & L & M & SI \\
\hline Yp & L & M & S & S & M & L \\
\hline
\end{tabular}

\section{PERFORMANCE EVALUATION}

In this subsection, the efficiency of the proposed solutions is examined through set of experiments. The first set of 
experiments is related to the proposed $G A$ while the second set of experiments is related to the adaptive fuzzy logic solution. The third type of experiments examines the effect of varying number of robots on the detection scheme especially in large-scale sensor networks.

\subsection{Simulation Environment}

The problem that was tackled in this section is to find a single radiation source in a certain space $A$. Based on the proposed simulation written in Matlab, considering the monitored field $A$ fixed within all of the experiments in this section, with a length 400 and width 400 distance units. The field is divided into 100 zones where the dimension of each zone is equal to 40 units. Each zone represents a subarea of the monitored field $A$. However, the area $A$ could be divided into a grid of zones with other dimensions. $A$ is also assumed to contain a dangerous hazard source in unknown location $\left(x_{s}, y_{s}\right)$ where $s$ refers to the hazardous source. The robot is assumed initially deployed at random position $\left(x_{i}, y_{i}\right)$. Robot is presumably having the capability of running different algorithms such as $G A$ and FL. It is also assumed to be capable of identifying its location from a priori given reference point. Moreover, it is assumed that the robot will have enough energy to complete its task. The algorithm for the source of hazard localization in specific area was divided into two phases. The first phase allows the robot to locally search in its zone to move from its start location and identifies the best location to move from the current zone to next one. This phase was accomplished using previously described GA solution. In the second phase of the algorithm, the robot is capable of specifying the right destination to move towards the next zone which mainly depends on its starting and ending points after running GA. Then, the robot moves to next zone and begins running GA again in the new zone until it reaches the source cell. At the algorithm ending, the remaining distance measurement between the robot and the hazardous source is possible.

\subsection{GA Based Experiments}

GA includes seeking and tuning of a number of specific parameters for optimizing its performance, namely number of generations, pop. size, and $P C$ and $P M$ percentages. Due to the stochastic nature of GA, the randomly generated initial population competence has a significant impact in the final performance of optimization. Thus, several trails were performed with different random initial populations. In addition, taking average results over the best solutions executed by each of parameter were used to draw the outline conclusions in this paper. Our experiments, in this section, start by testing the convergence of GA followed by defining the optimal number of population size. Another set of experiments are carried out to test the effect of both crossover and mutation on the objective function. Finally, another set of experiments are designed to study the effect of subareas dimension on the robot performance.

\subsubsection{Genetic Algorithm Convergence}

The present part of work is devoted for studying the changing the GA parameters effect on the remaining distance. In this set of experiments, the dependence of GA convergence speed on the number of generations is examined. A large number of experiments are conducted and the average results are presented in Fig. 3. as evidenced, the remaining distance is depicted against the number of generations till the algorithm is converged. Again, the value of the remaining distance is considered as an indicator to the detection scheme quality where best robot position is considered to be the nearest location to the source. In general, increasing generations resulted in convergence at a better robot location. Based on the average results (where the experiment was replicated 10 times), it seems that the GA converge after 100 generations. It is also worth mentioning that the remaining distance decreases slowly after generation number 200 . In terms of running time, the algorithm produces its results in few seconds which can be neglected. As a result, the default value of number of generations could be adjusted to 100 for next experiments.

\subsubsection{Optimal Number of Population Size}

Another set of experiments were carried out to specify the proper pop. size. Fig. 4 illustrates the value of the remaining distance versus the chromosomes number in the pop. size. The monitored field has the same characteristics as described in the previous subsection with different problem settings. As can be seen in the figure, different population size has been used in these experiments starting from 4 to 100 chromosomes per trail. However, the average best performance was accomplished with 20 chromosomes while for higher population size up to 100 , the rate of remaining distance decay was shown to be insignificant. This number is turned out to be effective since it saves the memory footprint required for the GA as well.

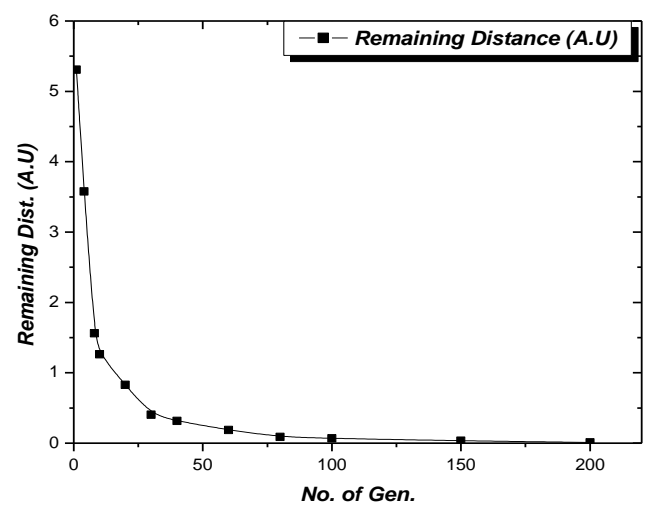

Fig. (3): Remaining distance as a Function of No. of generations

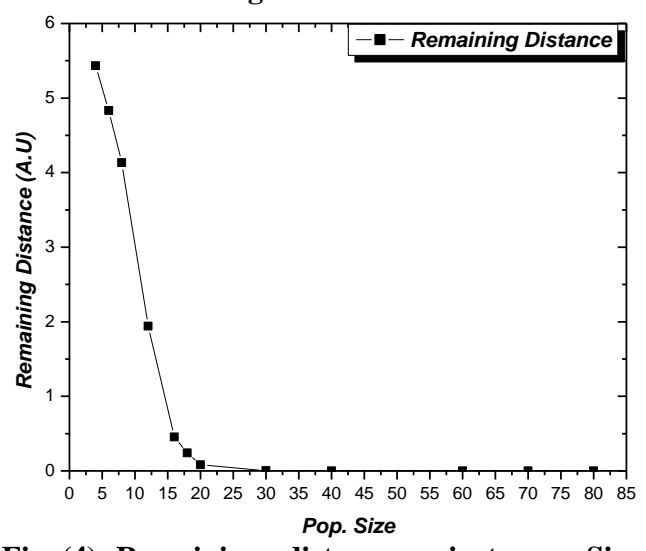

Fig. (4): Remaining distance against pop. Size 


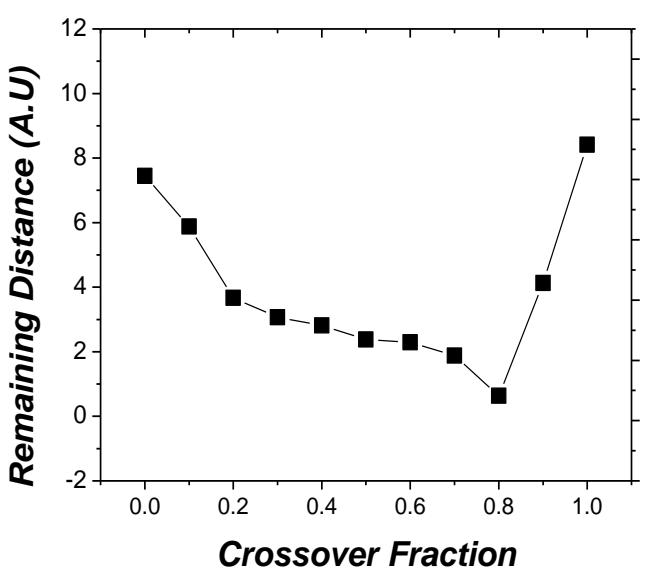

Fig. (5): Crossover fraction effect on remaining distance.

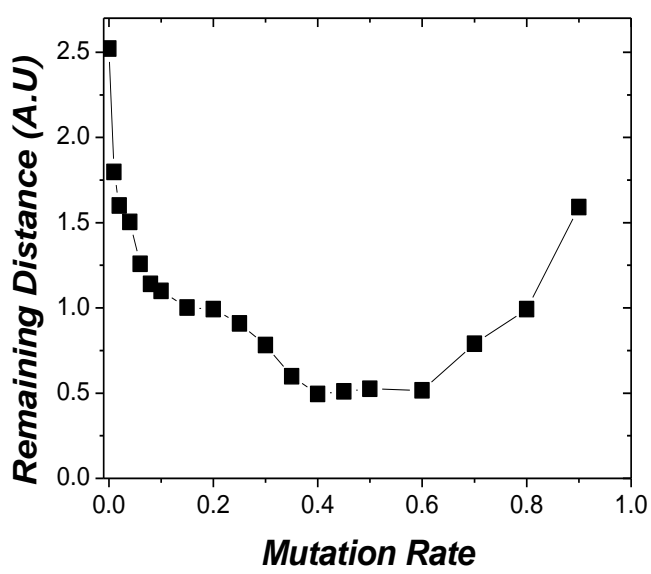

Fig. (6): Mutation rate effect on remaining distance

\subsubsection{Effect of Crossover Percentage on the} Remaining Distance

As an extension to the previous experiments, in this subsection, the algorithms illustrate the effect of changing crossover percentage $(P C)$ on the remaining distance progress as displayed in Fig. 5. The crossover percentage is designed to be changed from $0 \%$ up to $100 \%$. Based on the average collected results, it is found that, for the higher crossover percentage, the performance of the GA is dropped by increasing the fitness values. However and surprisingly, with $90 \%$ and more change in the crossover percentage, a severe increase in the fitness values is noticed. As a result, throughout the proposed experiments in this paper, the default value crossover percentage is set to $80 \%$.

\subsubsection{Effect of Mutation Percentage on the Objective Function}

Although the mutation could be an optional process in GA, it has been found that mutation percentage could have crucial effect in enhancing the performance of robot motion accuracy. Accordingly, the study was extended to include the effect of mutation percentage $\mathrm{Pm}$ which changes from $0 \%$ to $100 \%$ on remaining distance as illustrated in Fig.(6). It is clearly indicated that the Rate factor of 0.4 gives the best performance.

\subsubsection{Effect of Subarea Dimension on the Objective Function}

The effect of subarea dimension on the remaining distance is studied. Fig.(7) represents the dependence of remaining distance on subarea dimension. It is noticed that, as subarea dimension increases from 2 units up to 40 units, the remaining distance is slightly increased; on the other hand, the total distance of robot track decreases. For higher subarea dimension up to 100 , the rapid increase in the remaining distance was recorded. Further increase in subarea dimension up to 200 (A.U), the remaining distance slightly increases. As a consequence, it is appropriate for subarea dimension equals to 40 (A.U) to be the default value.

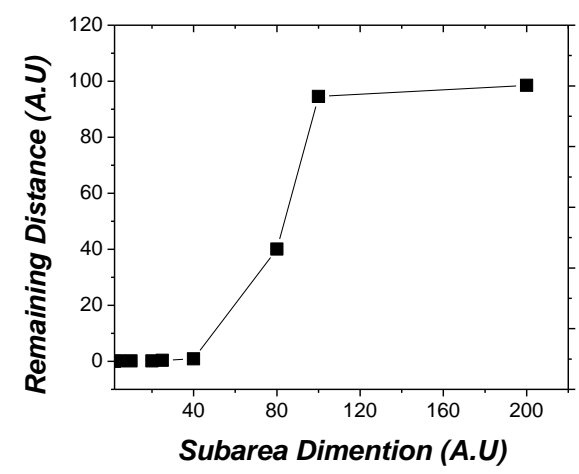

Fig. (7): Dependence of remaining distance on different values of subarea dimension using GA.

Applying all the above GA parameters settings, and considering the robot that is initially deployed at random position $\left(x_{i}, y_{i}\right)$ indicated with blue node, then the final representation of track, indicated with red line, that the robot follows to localize the radiological source (which is indicated with pink node) in the proposed monitored area, is displayed in Fig. (8). In addition, an example of robot walk using GA inside a zone after one generation is displayed in left side of the figure.

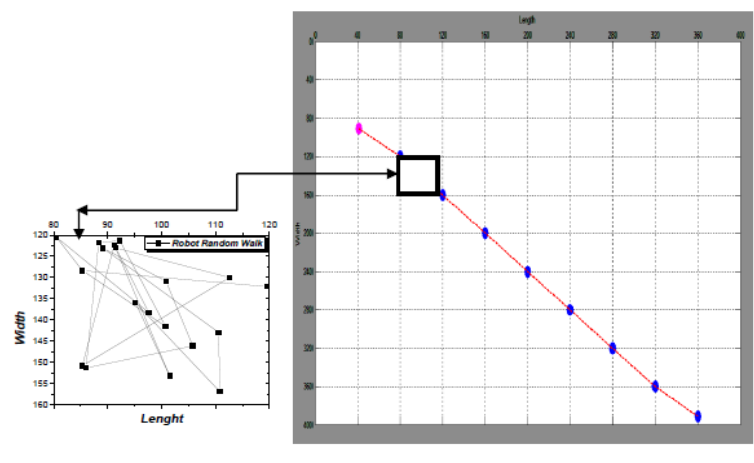

Fig. (8): Single robot track in the considered area

\subsubsection{Fuzzy based Self-Adaptive Simulation vs. GA Results}

The purpose of this section is presented the effect of both the optimizing capability and the convergence speed using fuzzy mechanism by self-adaptation of GAs parameters $\left(P_{C} \& P_{M}\right)$. The robot performance was tested at different generation values as shown in Fig. (9). The figure shows the comparison results of the GA and adaptive GA when different numbers of 
generation. It is found that, the adaptive GA algorithm outperforms regular GA by decreasing the number of generations where the best result was recorded at 30 generations instead of 100 when running GA.

The adaptive GA performance associated with using different number of chromosomes is also studied as plotted in Fig. (10). It is noted that, the adaptive GA proves to be more efficient as it decreases the population size to 12 instead of 20 when using GA.

Finally, the impact of subarea dimension on the remaining distance was investigated. It is noticed that, as displayed in

Fig.(11), there is no pronounced changes in the results obtained using adaptive GA when compared with those acquired using GA. So, it is appropriate for subarea dimension to be adapted at the same value

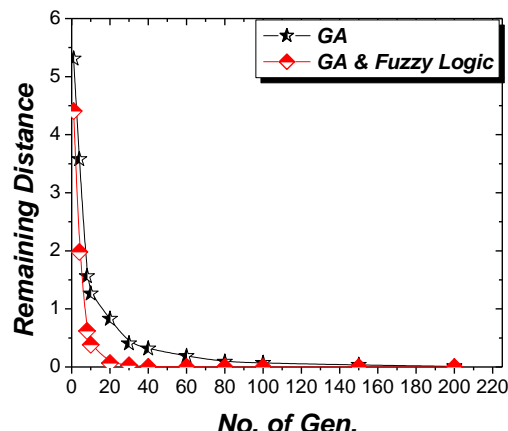

Fig. (9): GA and adaptive GA performance progress with number of generations.

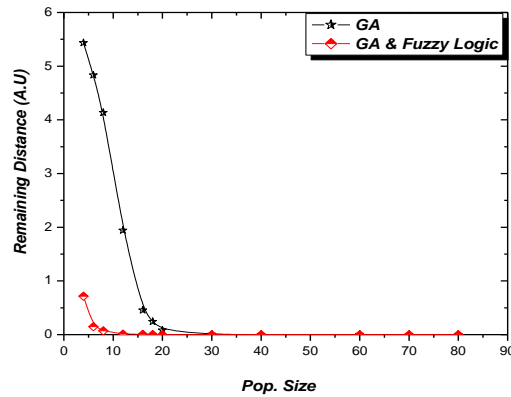

Fig. (10): Remaining Distance progress for GA and adaptive GA at different pop. Size.

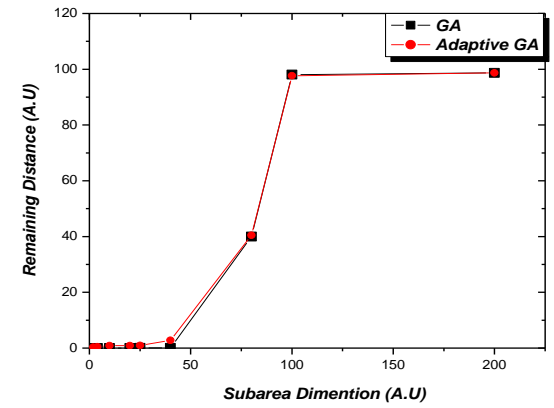

Fig. (11): GA and adaptive GA performance progress with different subarea dimension.
It could be clearly noted that, compared with GA, the optimizing capability and convergence speed of adaptive GA are largely improved by decreasing the number of generations which are reduced with percentage of $70 \%$. In addition, the population size is affected positively when running adaptive GA by reducing its value with a percentage of $40 \%$ rather than running GA. On the other hand, there is no significant change in subarea dimension value.

\subsection{Multiple Mobile Robots Behavior for Source Localization}

Figure (12) shows the tracks of 7 robots, deployed randomly in the proposed area when running LEAD algorithm to localize the radiation source, indicated with pink node. In addition, the paradigm of 7 robots tracks when running CONSLUT algorithm is presented in Fig. (13).

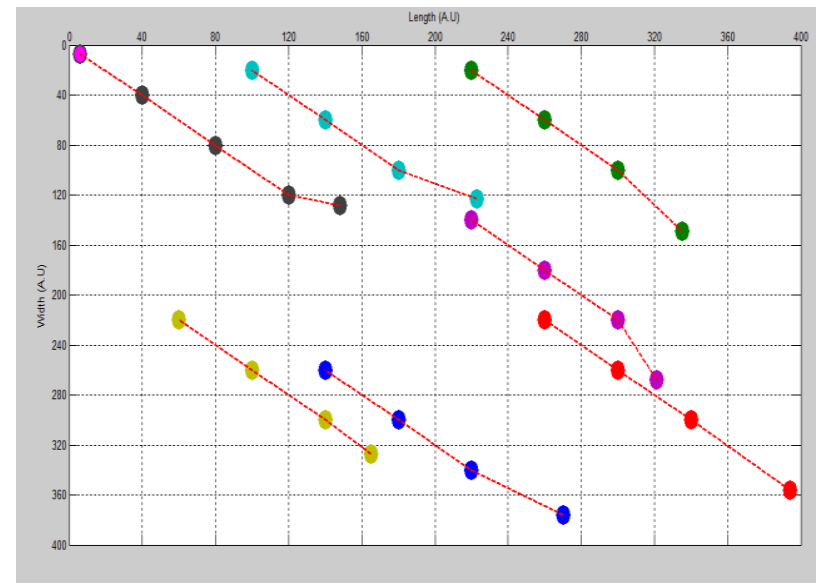

Fig. (12): Multi robots tracks in the considered area when running LEAD algorithm.

This shape is a sympol for radiation source

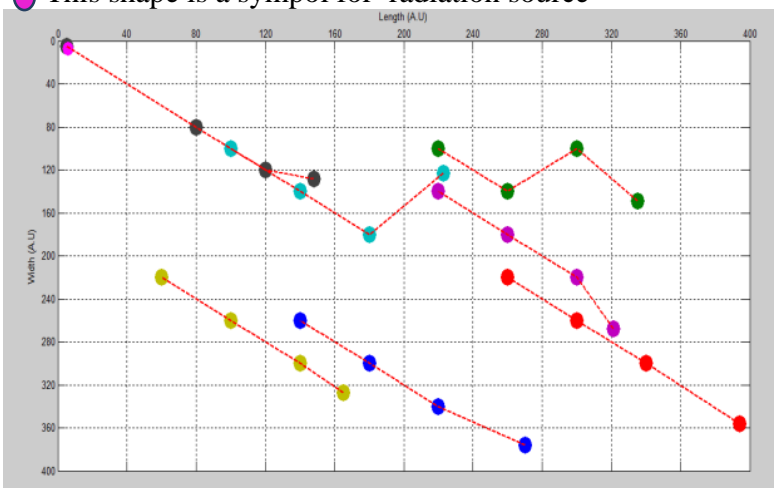

Fig. (13): Multi robots tracks in the considered area when running consult algorithm. 
For more efficient comparison results between two algorithms, the performance of both algorithms is tested by varying the radiation source location at different positions in the proposed area. Multiple snapshots were taken, for example, for different source locations when using 7 cooperative robots - for example - as being cleared in Fig. $(14 \mathrm{a}, \mathrm{b}, \mathrm{c})$. Moreover, the effect of varying robots number was taken in consideration where the same steps were repeated for different number of robots. In addition, Fig. (15 $\mathrm{d}, \mathrm{e}, \mathrm{f})$ displays different snapshots when using 2, 4 and 7 cooperative robots as an example. Finally, the average results are taken in consideration.

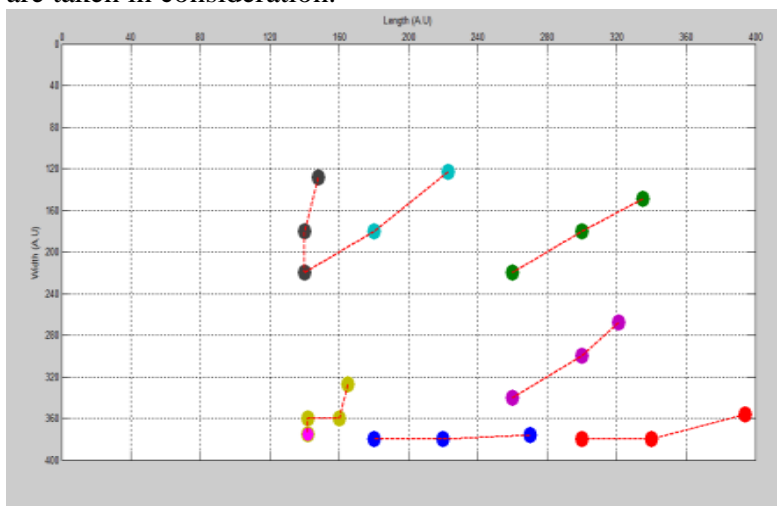

(a)

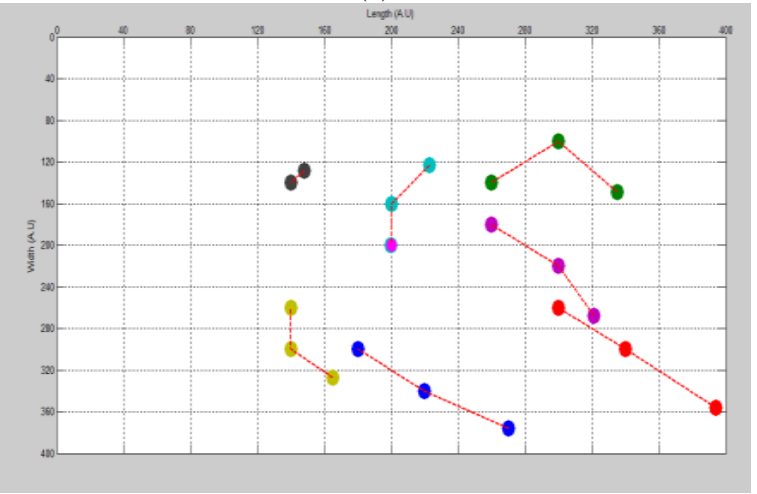

(b)

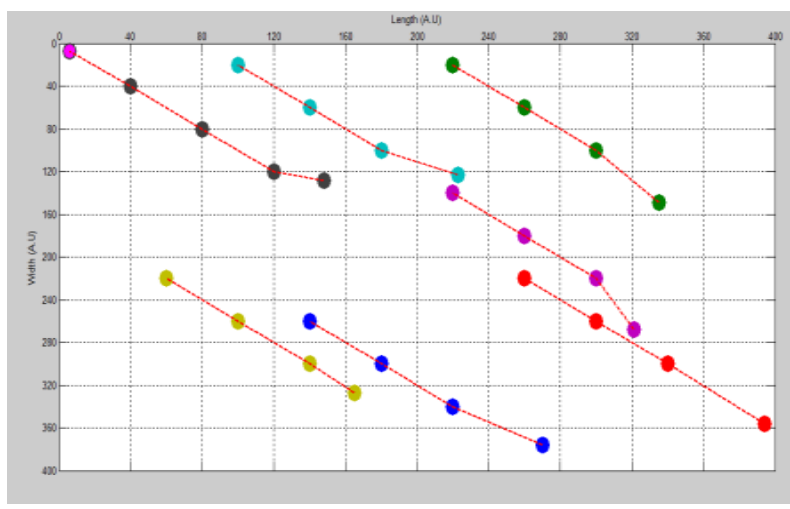

(c)

Fig. (14): Different Snapshots for different source locations (a, b, c)

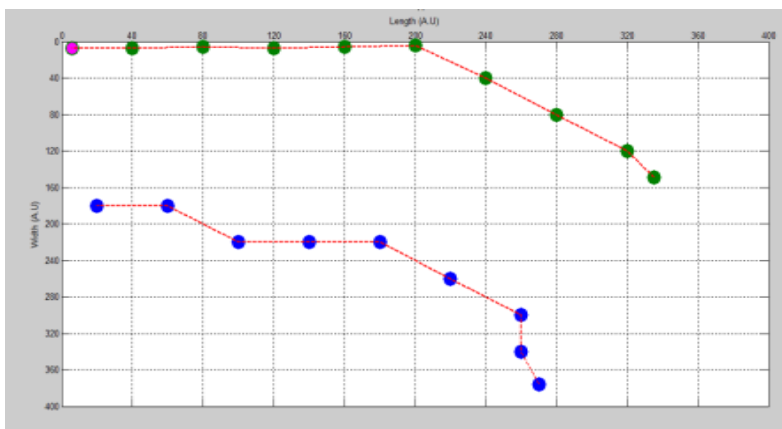

(a)

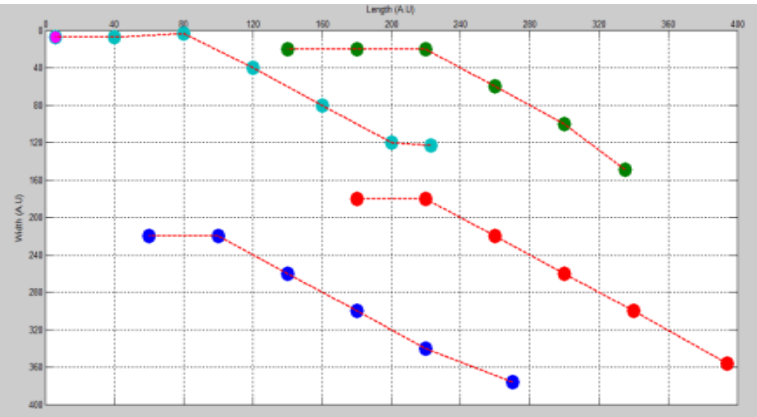

(b)

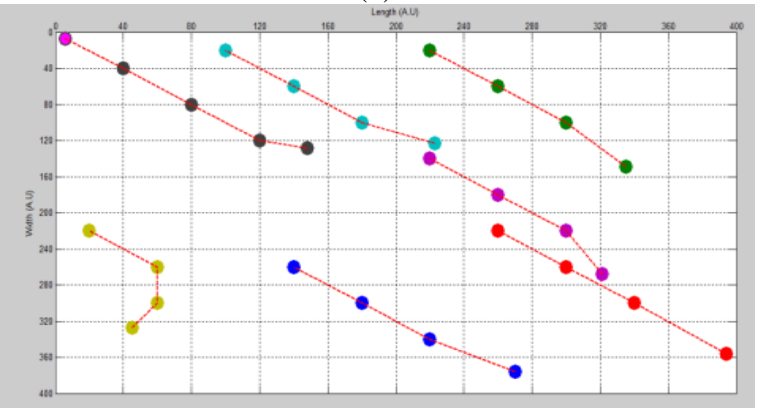

(c)

Fig. (15): Different Snapshots for different number of ropots locating the source $(a, b, c)$

As a consequence, the performance of both algorithms was tested under the previous explained conditions of different source positions for each number of robots where, in each case, the total distance of the considered robots that walked till any one of them could localize the hazard source is calculated. Finally, the comparison results are displayed in Fig. (16).

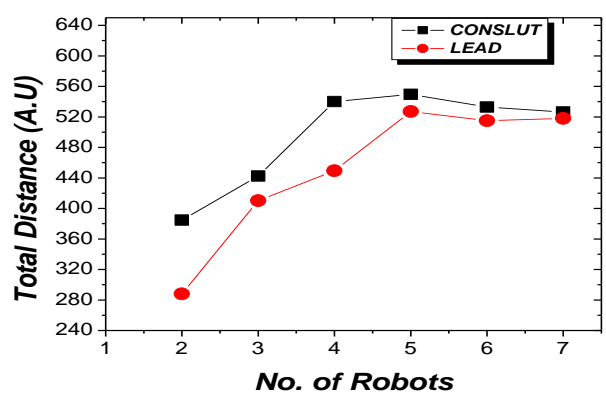

Fig. (15): LEAD and CONSULT algorithms performance progress with number of robots. 
It is found that, the LEAD algorithm outperforms CONSULT algorithm, by decreasing the total distance moved by the robots which is an indication of saving robot energy and wasted time. Also, it is worth mentioning that the new dimension in energy conservation has been added in favour of LEAD algorithm when considering the messages number that the $n$ robots send or receive for each hop. The LEAD algorithm seems to use fewer messages than CONSULT algorithm.

\section{CONCLUSION}

A novel algorithm based on GA is proposed for hazard radiation source localization problem using single robot. The adaptive GA based on fuzzy logic is also performed for comparison. Simulation results indicated that the proposed adaptive GA performs better and faster than GA by decreasing the number of generations which are reduced with percentage of $70 \%$. In addition, the population size is affected positively when running adaptive GA by reducing its value with a percentage of $40 \%$ rather than running GA. In addition, the performance of two novel algorithms (LEAD and CONSULT) assumed when considering group of autonomous mobile robots systems were compared. The LEAD algorithm outperforms CONSULT algorithm, by decreasing both the number of messages and total distance moved by the robots which is an indication of saving robot energy and wasted time. Simulation results showed that the robots could asymptotically approach and finally determine the odor source.

\section{REFERENCES}

[1] Jren-Chit Chin, et. al. "Identification of Low-Level Point Radioactive Sources Using a Sensor Network “, ACM Transactions on Sensor Networks, 7( 3), 2010, Article 21.

[2] Alan L. Melnick, "Biological, Chemical, and Radiological Terrorism Emergency Preparedness and Response for the Primary Care Physician", Springer, USA, 2008.

[3] John Fay, "Encyclopedia of security management", $2^{\text {nd }}$ edition, USA, 2007.

[4] Chee-Yee Chong and Srikanta P. Kumar, " Sensor Networks: Evolution, Opportunities, and Challenges", IEEE, 91( 8), 2003, pp. 1247-1256.

[5] Nor Azlina Ab. Aziz, Kamarulzaman Ab. Aziz, and Wan Zakiah Wan Ismail, "Coverage Strategies for Wireless Sensor Networks", World Academy of Science, Engineering and Technology, 50, 2009, 145-150.

[6] You-Chiun Wang, Chun-Chi Hu, and Yu-Chee Tseng," Efficient Placement and Dispatch of Sensors in a Wireless Sensor Network", IEEE Transactions On Mobile Computing, 7 (2), 2008, 262 - 274.

[7] Peter Csaba Ölveczky and Stian Thorvaldsen, "Formal modeling, performance estimation, and model checking of wireless sensor network algorithms in Real-Time Maude", Theoretical Computer Science, 410, 2009, 254280.

[8] Ajith Gunatilaka, et. al, "Radiological Source Localisation", Defence Science and Technology Organisation, Commonwealth of Australia, 2007.

[9] Chandy, M. et. al, "Networked sensing systems for detecting people carrying radioactive material",
International Conference on Networked Sensing Systems, IEEE, 2008, 148-155.

[10] Annie Liu, "Simulation and Implementation of Distributed Sensor Network for Radiation Detection", Thesis In Partial Fulfillment of the Requirements for the Degree of Master of Science, California Institute of Technology Pasadena, California, 2010.

[11] Qing Mao, Tiehui Li, Ping Dong, Ke Ding, and Tinghuai Ma, "Sensor Grid-Based Radiation Detection System in High Energy Physics" Third International Conference on,Convergence and Hybrid Information Technology, 2008. ICCIT '08. 1, 11-13 Nov. 2008, 830-836.

[12] Cheng Xiang-jin, Shi Yi-kai, Yang Ning, and Huang Lei, "Design of radiation detection system with WSN," Cross Strait Quad-Regional Radio Science and Wireless Technology Conference (CSQRWC), 26-30 July 2011, 946-949.

[13] Qing-Hao Meng et. al, "Mobile Robots Odor Localization with an Improved Ant Colony Algorithm", Robotics and Biomimetics, ROBIO '06. IEEE International Conference, 2006, 959 - 964 .

[14] A. Hayes, A. Martinoli, and R.M.Goodman, "Distributed Odor Source Localization", IEEE Sensors Journal, Special Issue on Electronic Nose Technologies, 2( 3), 2002, 260-271.

[15] A. Hayes, A. Martinoli, and R.M.Goodman, "Swarm Robotic Odor Localization: Off-Line Optimization and Validation with Real Robots", Robotica, 21( 4), 2003, 427-441.

[16] A. Hayes, A. Martinoli, and R.M.Goodman. Odor Source Localization with Mobile Robots.

Bulletin of the Swiss Society for Automatic Control, 46, 2007, 11-14.

[17] Jren-Chit et. al, "Accurate Localization of Low-level Radioactive Source Under Noise and Measurement Errors", SenSys'08 Proceedings of the 6th ACM conference on Embedded network sensor systems, New York, NY, USA, 2008, 183-196.

[18] Colin R.Reeves and Jonathan E.Rowe, "Genetic Algorithms: Principles and Perspective A Guide to GA Theory“, Kluwer Academic Publishers, Dordrecht, 2003.

[19] Julian F. Miller, "Cartesian Genetic Programming", Springer-Verlag Berlin Heidelberg, 2011.

[20] S. N. Sivanandam, S. Sumathi and S. N. Deepa, "Introduction to Fuzzy Logic using MATLAB", Springer-Verlag Berlin Heidelberg, 2007.

[21] Guanrong Chen and Trung Tat Pham, "Introduction to Fuzzy Sets, Fuzzy Logic, and Fuzzy Control Systems", CRC Press LLC United States of America, 2001.

[22] Dun-wei Gong et. al, "Modified Particle Swarm Optimization for Odor Source Localization of Multirobot", Evolutionary Computation (CEC), IEEE Congress, 2011, 130 - 136. 